The time-averaged pulsed flux density of the pulsars is about $1.8 \mathrm{mJy}\left(1 \mathrm{Jy}=10^{-26} \mathrm{~W} \mathrm{~m}^{-2}\right.$ $\mathrm{Hz}^{-1}$ ) at $1390 \mathrm{MHz}$ (Table 1), compared with a total flux density at this frequency of $7 \mathrm{mJy}$ (3). The $\sim 5$-mJy unpulsed emission probably arises in the impact region described above. We find it remarkable, with much of the magnetosphere of B blown away by the wind of $\mathrm{A}$, that $\mathrm{B}$ still works as a pulsar. This suggests that the radio emission is probably generated close to the neutron star, providing a direct constraint on the emission height.

Conclusion. We have detected the binary companion of the millisecond pulsar J0737-3039 as a pulsar, making this the first known double-pulsar system. This discovery confirms the neutron-star nature of the companions to recycled pulsars in eccentric binary systems and validates the suggested evolutionary sequences in which a companion star, having spun up the pulsar, forms a young pulsar in a supernova explosion (31). The highly relativistic nature of this compact system opens up opportunities for much more stringent tests of relativistic gravitation than have been possible previously. Not only have we already measured four quantities attributable to, and consistent with, general relativity, but the mass ratio $R$ is a new high-precision constraint that is independent of gravitational theories. Within a year or so, we expect to measure the orbital decay due to emission of gravitational radiation. If the intrinsic value due to gravitational-wave damping can be extracted, it will allow tests of radiative aspects of gravitational theories mixed with strong-field effects. On somewhat longer time scales of a few years, we expect to detect several other relativistic effects, such as geodetic precession of the pulsars' spin axes, spinorbit coupling, and other deviations, making this a superb test bed for relativity.

The detection of the companion as a pulsar also opens up the possibility of using each pulsar to probe the magnetosphere of the other. The energy flux from the millisecond pulsar is strongly affecting the pulse emission process in the companion, and eclipses of the millisecond pulsar by the companion are also seen. Future measurements of orbital variations in pulse shapes, amplitudes, polarization, and timing over a range of radio frequencies will give fascinating insights into magnetospheric processes in pulsars.

\section{References and Notes}

1. J. H. Taylor, Philos. Trans. R. Soc. London Ser. A 341, 117 (1992).

2. R. A. Hulse, J. H. Taylor, Astrophys. J. 195, L51 (1975)

3. M. Burgay et al., Nature 426, 531 (2003).

4. V. Kalogera et al., Astrophys. J., in press; preprint is available at http://arxiv.org/abs/astro-ph/0312101.

5. Receiver bandwidths used were $64 \mathrm{MHz}$ at $680 \mathrm{MHz}$, $256 \mathrm{MHz}$ at $1390 \mathrm{MHz}$, and $576 \mathrm{MHz}$ at $3030 \mathrm{MHz}$.

6. More information is available at http://pulsar. princeton.edu/tempo/.

7. T. Damour, J. H. Taylor, Phys. Rev. D 45, 1840 (1992).

8. J. H. Taylor, J. M. Weisberg, Astrophys. J. 345, 434 (1989)
9. I. H. Stairs, S. E. Thorsett, J. H. Taylor, A. Wolszczan, Astrophys. J. 581, 501 (2002).

10. M. Bailes, S. M. Ord, H. S. Knight, A. W. Hotan, Astrophys. J. 595, L49 (2003).

11. T. Damour, N. Deruelle, Ann. Inst. Henri Poincaré 44, 263 (1986).

12. T. Damour, J. H. Taylor, Astrophys. J. 366, 501 (1991).

13. T. Damour, N. Deruelle, Ann. Inst. Henri Poincaré 43, 107 (1985).

14. This is correct up to so-called first post-Newtonian $\left[(v / c)^{2}\right]$ order (7), and any intersection of the PK-parameter lines must be located on the $\mathrm{R}$ line, which will only deviate from that shown in Fig. 3 by corrections of order $(v / c)^{4}$.

15. This is correct because of the usage of the DD timing model in our analysis and its definition of mass $(11,13)$.

16. T. Damour, R. Ruffini, C. R. Acad. Sci. Paris Ser. A 279, 971 (1974).

17. B. M. Barker, R. F. O'Connell, Astrophys. J. 199, L25 (1975).

18. J. M. Weisberg, R. W. Romani, J. H. Taylor, Astrophys. J. 347, 1030 (1989).

19. J. M. Weisberg, J. H. Taylor, Astrophys. J. 576, 942 (2002).

20. M. Kramer, Astrophys. J. 509, 856 (1998).

21. T. Damour, G. Schäfer, Nuovo Cimento 101, 127 (1988).

22. N. Wex, Class. Quantum Gravity 12, 983 (1995).

23. D. Bhattacharya, E. P. J. van den Heuvel, Phys. Rep. 203, 1 (1991)

24. G. S. Bisnovatyi-Kogan, B. V. Komberg, Sov. Astron 18, 217 (1974).

25. N. Shibazaki, T. Murakami, J. Shaham, K. Nomoto, Nature 342, 656 (1989).

26. Z. Arzoumanian, J. M. Cordes, I. Wasserman, Astrophys. J. 520, 696 (1999).
27. E. P. J. van den Heuvel, C. de Loore, Astron. Astrophys. 25, 387 (1973).

28. B. P. Flannery, E. P. J. van den Heuvel, Astron. Astrophys. 39, 61 (1975).

29. S. E. Thorsett, D. Chakrabarty, Astrophys. J. 512, 288 (1999).

30. R. N. Manchester, J. H. Taylor, Pulsars (Freeman, San Francisco, 1977).

31. G. Srinivasan, E. P. J. van den Heuvel, Astron. Astrophys. 108, 143 (1982).

32. J. H. Taylor, J. M. Cordes, Astrophys. J. 411, 674 (1993).

33. We would like to thank J. Sarkissian and other members of the Parkes multibeam team for their kind help with making the observations described in this paper and $\mathrm{N}$. Wex and G. Schäfer for useful discussions. Extensive use was made of the PSRCHIVE pulsar analysis system developed by A. Hotan and colleagues (see http:// astronomy.swin.edu.au/pulsar). The Parkes radio telescope is part of the Australia Telescope, which is funded by the Commonwealth of Australia for operation as a National Facility managed by CSIRO. M.B., A.P., and N.D. acknowledge financial support from the Italian Ministry of University and Research (MIUR) under the national program Cofin 2001. F.C. is supported by NSF, NASA, and National Radio Astronomy Observatory. D.R.L. is a University Research Fellow funded by the Royal Society.

12 December 2003; accepted 30 December 2003 Published online 8 January 2004; $10.1126 /$ science. 1094645

Include this information when citing this paper.

\title{
Empathy for Pain Involves the Affective but not Sensory Components of Pain
}

\author{
Tania Singer, ${ }^{1 *}$ Ben Seymour, ${ }^{1}$ John O'Doherty, ${ }^{1}$ Holger Kaube, ${ }^{2}$ \\ Raymond J. Dolan, ${ }^{1}$ Chris D. Frith ${ }^{1}$
}

\begin{abstract}
Our ability to have an experience of another's pain is characteristic of empathy. Using functional imaging, we assessed brain activity while volunteers experienced a painful stimulus and compared it to that elicited when they observed a signal indicating that their loved one-present in the same room-was receiving a similar pain stimulus. Bilateral anterior insula (AI), rostral anterior cingulate cortex (ACC), brainstem, and cerebellum were activated when subjects received pain and also by a signal that a loved one experienced pain. Al and ACC activation correlated with individual empathy scores. Activity in the posterior insula/secondary somatosensory cortex, the sensorimotor cortex (SI/MI), and the caudal ACC was specific to receiving pain. Thus, a neural response in $\mathrm{Al}$ and rostral ACC, activated in common for "self" and "other" conditions, suggests that the neural substrate for empathic experience does not involve the entire "pain matrix." We conclude that only that part of the pain network associated with its affective qualities, but not its sensory qualities, mediates empathy.
\end{abstract}

Human survival depends on the ability to function effectively within a social context. Central to successful social interaction is the ability to understand others intentions and beliefs. This capacity to represent mental states is referred to as "theory of mind"

${ }^{1}$ Wellcome Department of Imaging Neuroscience, Institute of Neurology, University College of London, 12 Queen Square, WC1N 3AR London, UK. ${ }^{2}$ Headache Group, Institute of Neurology, University College of London, Queen Square, WC1N 3BG London, UK.

*To whom correspondence should be addressed. Email: t.singer@fil.ion.ucl.ac.uk
(1) or the ability to "mentalize" (2). Empathy, by contrast, broadly refers to being able to understand what others feel, be it an emotion or a sensory state. Accordingly, empathic experience enables us to understand what it feels like when someone else experiences sadness or happiness, and also pain, touch, or tickling (3).

Even though empathy has been extensively discussed and investigated by philosophers and social scientists, only recently has it become a focus for neuroscience (3-8). Influenced by perception-action models of motor behavior and imitation (9), 
Preston and de Waal (8) proposed a model of empathy that incorporates most theoretical accounts of, as well as empirical findings on, empathy. The key suggestion is that observation or imagination of another person in a particular emotional state automatically activates a representation of that state in the observer, with its associated autonomic and somatic responses ("automatic" refers to a process that does not require conscious and effortful processing but can nevertheless be inhibited or controlled). The philosopher Susanne Langer has described it as an involuntary breach of individual separateness (10).

Recent neuroimaging studies have explored the neural correlates of empathic experience by measuring brain activity while subjects watched video clips showing actors telling personal stories with neutral or sad content accompanied by sad and happy facial expressions (11), by comparing brain activity associated with the imitation of and the mere observation of pictures showing different emotional facial expressions (12), and by comparing neural responses elicited by watching videos of faces with emotional expressions of disgust and pleasure with responses induced by smelling aversive and pleasant odors (13). Whereas the first study did not permit the identification of shared networks underlying emotions in self and others, the latter studies report activation in areas previously identified in the perception and production of facial emotional expressions (action representation and emotional systems) as well as during the smelling of aversive odors (insula). These results suggest that regions associated with feeling an emotion can be activated by seeing the facial expression of the same emotion, a phenomenon described as emotional contagion.

A paradigm to study empathy in vivo. The present study extends previous findings in a number of ways. First, we tested for shared and unique networks for experienced and empathic pain. More specifically, we provide evidence for painrelated empathic responses and demonstrate that empathic experience does not involve activation of an entire pain matrix, but only of that component associated with the affective dimension of pain experience.
Fig. 1. Pain-related activation associated with either experiencing pain in oneself or observing one's partner feeling pain. Areas in green represent significant activation $(P<0.001)$ for the contrast pain-no pain in the "self" condition and areas in red for the contrast pain-no pain in the "other" condition. The results are superimposed on a mean structural scan of the 16 subjects. Activations are shown on sagittal (A and $B$ ) and axial (C and D) slices. (A) Activation in ACC and cerebellum. (B) Bilateral insula cortex extending into lateral prefrontal cortex, left posterior insula extending into secondary somatosensory cortex (SII), bilateral occipital cortex, and fusiform cortex. (C) Bilateral insula and mediodorsal thalamus. (D) Middle and lateral cerebellum/ fusiform gyrus. For coordinates of peak activations from "self" and "other" conditions, see tables S1 and S2.

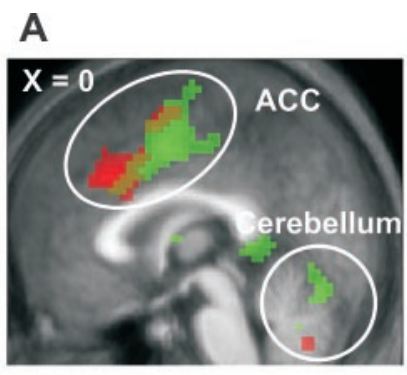

$B$
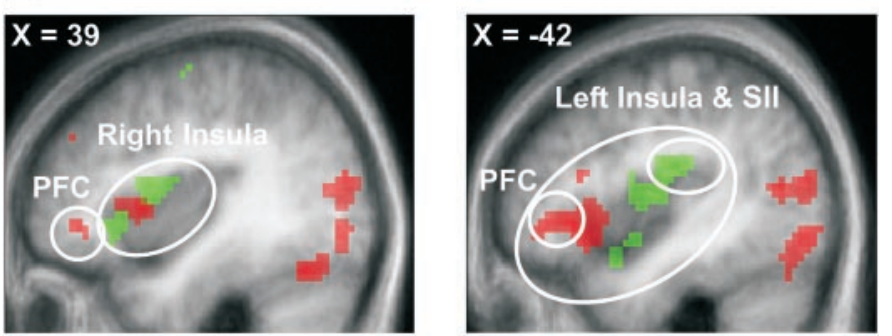

C

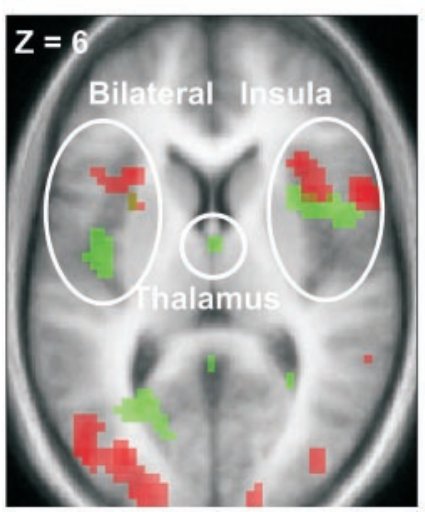

Moreover, we show a relation between empathy-related brain activity and individual difference in empathy as assessed by commonly used empathy scales. We also demonstrate - in contrast to accounts of emotional contagion-that empathic responses can be elicited automatically in the absence of an emotional cue (such as facial emotional expressions) through mere presentation of an arbitrary cue that signals the feeling state of another person.

We used functional magnetic resonance imaging (fMRI) to explore both common and unique brain circuitry involved when we experience pain in ourselves, as well as when we observe someone else feeling pain. Previous studies on pain have revealed that noxious stimuli consistently activate the secondary somatosensory cortex (SII), insular regions, the anterior cingulate cortex (ACC), the movement-related areas such as the cerebellum and supplementary motor areas and, less robustly, the thalamus and the primary somatosensory cortex (SI) $(14,15)$. This pain-related network, which is commonly referred to as the pain matrix, served to define regions of interest in the present study.

To investigate pain-related empathy, we developed a paradigm that allows the investigation of empathic experience in vivo, with the object of empathy being a real person present in the same room. More specifically, we investigated pain-related empathy in 16 couples, under an assumption that couples are likely to feel empathy for each other. We assessed brain activity in the female partner while painful stimulation was applied to her or to her partner's right hand through an electrode attached to the back of the hand. The partner was seated next to the MRI scanner and the right hand of each subject was placed on a tilted board, allowing the female partner with help of a mirror system to see her and her partner's right hand. On a large screen situated behind the board, cues were presented in random order indicating whether she (self) or her partner (other) would get low (no pain condition) or high (pain condition) stimulation. We were especially interested in comparing pain-related brain activity (assessed by the difference between trials involving painful and nonpainful stimulation) in the context of "self" and "other." Questionnaires administered after scanning served to validate measurements of individual pain threshold made before scanning, to obtain subjective evidence for empathic experience during scanning, and to assess stable individual differences in empathy in order to determine whether these scores predict the amplitude of empathy-related brain activity.

Analysis of pain intensity ratings after 
scanning confirmed the individual thresholds for nonpainful and painful stimulation determined before scanning (fig. S1) (16). In addition, the unpleasantness ratings indicated empathic involvement of the subjects. Nonpainful trials were rated as being significantly less unpleasant than painful trials, irrespective of whether the pain was applied to themselves or to the partner [main effect of pain: $\mathrm{F}(1,15)=19.93, P<$ 0.001 ; main effect of self/other: $\mathrm{F}(1,15)=$ $0.12, P=0.73)]$.

Does empathizing with pain in others activate the entire pain matrix? Comparison of brain activity associated with painful and nonpainful trials in the "self" condition revealed increased activity in contralateral SI/MI, in bilateral SII with a peak activation in contralateral posterior insula extending into SII, in bilateral mid and anterior insula, in ACC [caudal and posterior rostral zones, using Picard and Strick's terminology, (17)], in right ventrolateral and mediodorsal thalamus, brainstem, and mid and right lateral cerebellum (Fig. 1, A to D, green). These regions have all been identified as responding to painful stimuli in previous imaging studies $(14,15)$. Many structures in this pain matrix were also activated when pain was applied to the partner, that is, in the absence of somatosensory stimulation (Fig. 1, A to D, red). When comparing painful with nonpainful trials in the context of "other," increases in brain activity were observed in the ACC (anterior and posterior rostral zones), the anterior insula (AI) bilaterally with an extension into inferior prefrontal cortex, the cerebellum, and the brainstem. In addition, significant activations were observed in the ventral and dorsal visual stream, including bilateral fusiform cortex, lateral occipital and right posterior superior temporal sulcus, the left inferior parietal cortex, and the left superior frontal cortex.

To test for common networks activated by pain in the self and other conditions, we performed a conjunction analysis (Fig. 2, A and $\mathrm{B}$ ), as well as a more conservative masking procedure in which we masked the simple contrast pain-no pain in others by the simple contrast pain-no pain in self. Both analyses revealed a network common to pain in self and other conditions that comprised ACC (caudal and posterior rostral zones), bilateral middle insula and $\mathrm{AI}$ (with a peak activation in the right $\mathrm{AI}$ ), brainstem, and lateral cerebellum.

We also tested for an interaction in order to identify regions where there were stronger pain-related activations in the self as compared to the other condition. These analyses confirmed that the contralateral activations in SI/MI and SII/posterior insula, as well as a region in caudal ACC, were specific to the pain experience in the self condition (Fig. 3). In contrast, extrastriate visual related activations were specific to the empathy condition.

Inspection of the time courses of painrelated activity for self and others suggest two peaks, probably as the result of an instant response to the anticipation cue followed by another response to the delivery of the pain stimulus $3.5 \mathrm{~s}$ later. Accordingly, pain-related activations for self in ACC and AI peak first early, around 2 to $4 \mathrm{~s}$, and again around 8 to $12 \mathrm{~s}$ (Fig. 2C). Activation specific to pain in self in SI, SII, and caudal ACC seems only to peak late, around 8 to $12 \mathrm{~s}$, probably registering the actual receipt of the painful stimulus (Fig. 3, A to C). Although the present study was not designed to test differences between anticipation and receipt of pain in self and others, the present data suggest that both anticipation and experience of pain involve ACC and AI and that activation of these networks is involved in understanding the pain of others.

Do people scoring higher in empathy show higher pain-related brain activity? To

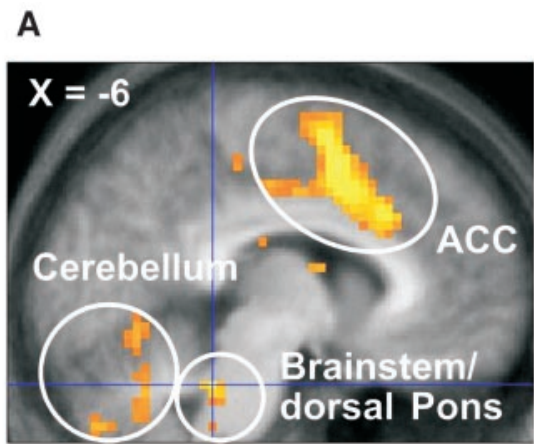

C

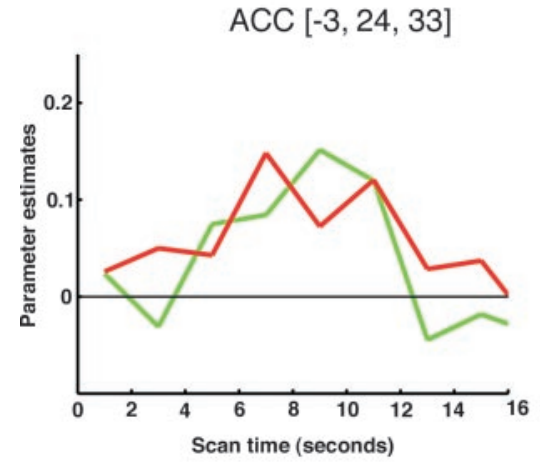

Right insula [39, 12, 3]

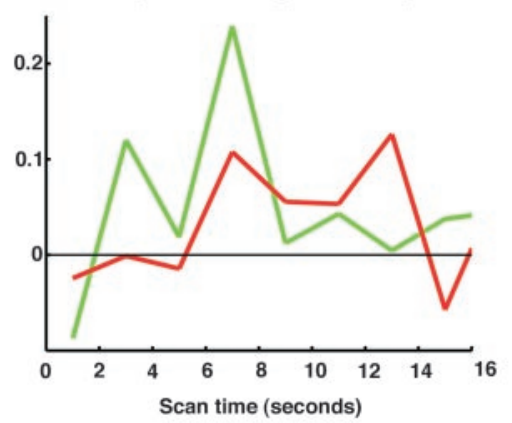

Fig. 2. Shared networks observed when pain was applied to self or to the partner. (A) and (B) illustrate results of a conjunction analysis between the contrasts pain-no pain in the context of self and other at $P<0.001$. Results are shown on sagittal (A) and coronal (B) sections of the mean structural scan. Coordinates refer to peak activations and are in $\mathrm{mm}$. Increased pain-related activation was observed in $\operatorname{ACC}[(-9,6,42) ;(0,24,33)]$, left insula $[(-30,9,6)$; $(-39,0,18) ;(-48,12,-3)]$, right anterior insula $[(60,15,3) ;(39,12,3) ;(42,27,-6)]$, cerebellum $[(-33,-66,-24) ;(27,-66,-18)]$ and brainstem $(-6,-39,-30)$. (C) The time courses of pain-related activation (pain-no pain) for peak activations in ACC and right anterior insula cortex for self (green lines) and others (red lines). A complete list of activated areas is in table S3. The anticipation cue was presented at time 0 , and the pain was applied between 3 and $4 \mathrm{~s}$ into the trial (mean $3.5 \mathrm{~s}$ ). 


\section{RESEAR C H AR T I C LES}

These findings underscore the crucial role of rostral ACC and anterior insula cortices for empathic experience related to pain. They are also in line with a report based on single-neuron recordings in a precingulotomy patient that provided evidence that neurons in the ACC can respond not only when a person receives a painful stimulus but also when a person observes or anticipates a potentially painful stimulus delivered to an experimenter (21). Note that these regions are fundamentally different from sites subserving mirror neurons that respond when performing or watching a conspecific performing particular movements (22).

Numerous imaging studies have investigated the neural correlates of pain experience $(14,15)$, but none have explored the empathic experience of pain. Our results confirm previous findings of pain-related activation in SI, SII, bilateral insula cortex, ACC, thalamus, brainstem, and cerebellum. In addition, our data show that only part of the network mediating pain experience is shared when empathizing with pain in others. Empathizing with someone else's pain elicited activity principally in left and right AI, ACC, lateral cerebellum, and brainstem. This activity was elicited without an explicit focus on empathy insofar as subjects were not aware of the study aims, nor were they required to make an explicit judgement of what their loved one was feeling at the time of the scan. The finding of empathy-related activation suggests an automatic engagement of empathic processes when perceiving pain in others. Moreover, our analysis demonstrates that pain-related activation in contralateral SI, SII/posterior insula, and caudal ACC are specific to self-experienced pain, as opposed to perceived pain in others.

Recent neuroimaging studies on pain have demonstrated contralaterally biased representations of painful stimulus in SI, distinct parts of SII, and posterior insula, as well as in lateral thalamus, which suggests that these structures provide sensorydiscriminative information concerning nociceptive stimuli such as location, quality, and intensity $(23,24)$. In contrast, AI and ACC do not show such a contralateral bias, supporting a role in coding the autonomic and affective dimension of pain such as the subjectively perceived unpleasantness (15, 25-27). Using hypnosis as a tool to disassociate sensory-discriminative from affective pain components, activation in $\mathrm{ACC}$ (posterior rostral zone) was shown to be modulated by perceived unpleasantness, whereas activation in SI and SII was unaffected (28). Similarly, attentional manipulations modulate ACC (posterior rostral zone) and right AI, but not posterior insular/ SII cortices (29). The role of ACC (posterior rostral zone) and right $\mathrm{AI}$ in coding the

\section{A}
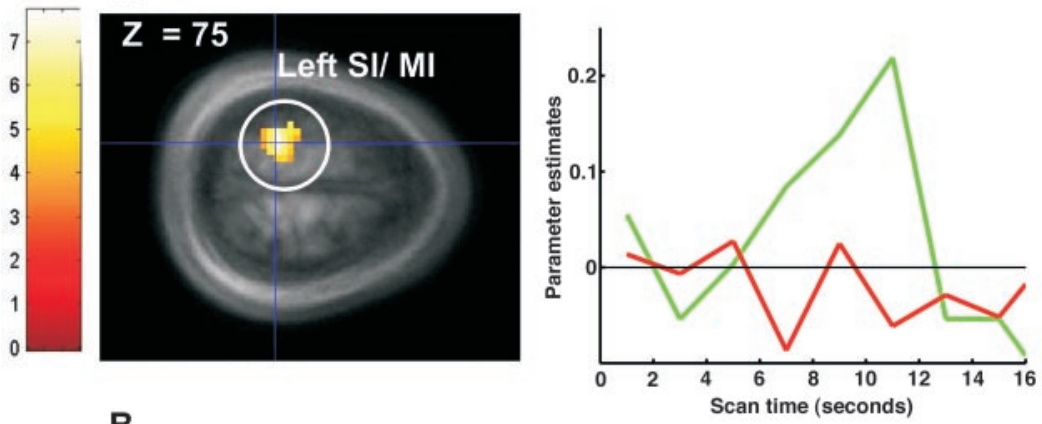

B
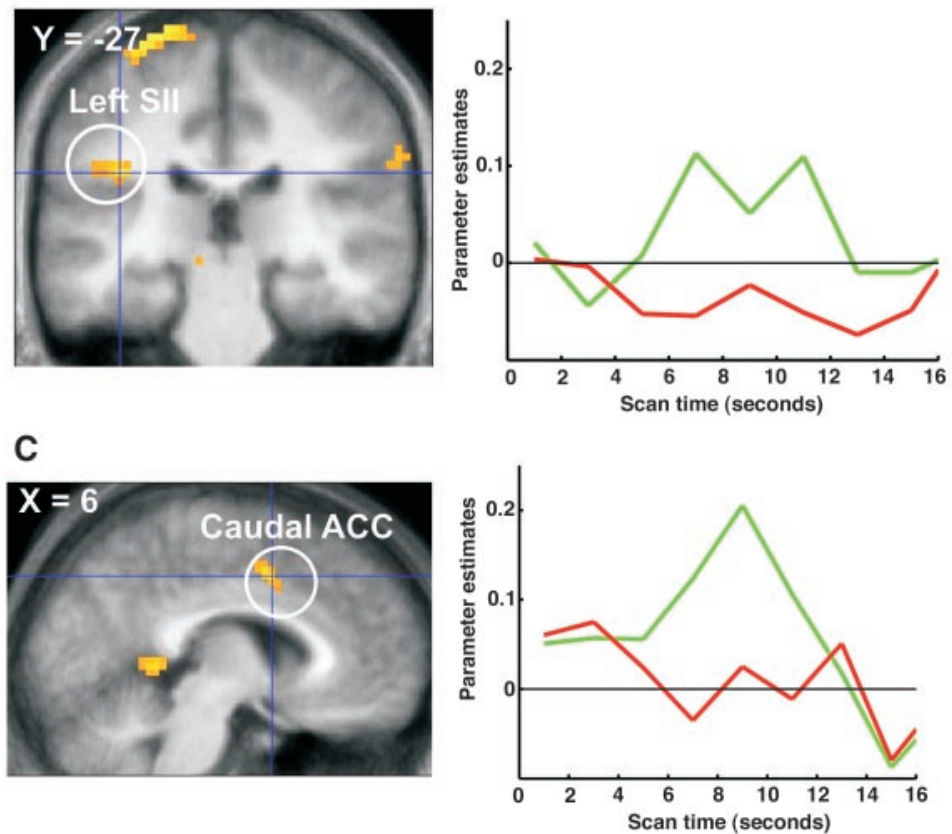

Fig. 3. Brain activity specific to the experience of pain in oneself. (A to $\mathbf{C}$ ) illustrate results of the interaction between the two factors (pain-no pain and self/other). Statistical maps (threshold set at $P<0.001$ ) are overlayed on axial $(A)$, coronal $(B)$, and sagittal $(C)$ sections from the mean structural scan. Coordinates refer to peak activations and are in $\mathrm{mm}$. (A) Increased pain-related activity $[(-21,-33,75) ;(-30,-24,72) ;(-27,-36,60)]$ in SI/MI. (B) Activity in left posterior insula/SII $[-39,-27,24]$. (C) Activity in a part of caudal ACC $(6,6,42)$. On the bottom, time courses are displayed for peak voxels of contralateral SI, SII, and caudal ACC. Green lines reflect parameter estimates for pain-related activity (pain-no pain) in self; red lines reflect pain-related activity in others.

subjective affective dimension of pain is also highlighted by findings showing that activation of right AI was correlated with subjective intensity ratings of thermal sensation in a manner that is distinct from a linear representation of stimulus temperature in posterior insula (30). Indeed, anticipation of pain activates more anterior insular regions, whereas the actual experience of pain activates more posterior insula, which suggests that the latter is associated with the actual sensory experience of pain and the former with affective dimensions such as the anticipatory arousal and anxiety of pain (31). Furthermore, subjective reduction of pain associated with placebo and opioid analgesia is associated with increased activity in rostral ACC and right AI (32).
Conclusion. The strong anatomical connections between regions constituting the pain matrix suggest that these regions do not function independently in encoding different aspects of pain but are highly interactive. Nevertheless, the results of the present study and previous investigations suggest a segregation of sensory-discriminative and autonomic-affective attributes of the pain experience. Rostral ACC and AI appear to reflect the emotional experience that evokes our reactions to pain and constitutes the neural basis for our understanding of the feelings of others and ourselves.

The above proposal is consistent with a recent model by Craig based on detailed anatomical observations that conceives of pain as one of the homeostatic emotions that reflects the internal (interoceptive) 
RESEARCH ART ICLES

A

Empathic Concern Scale (EC, DAVIS)
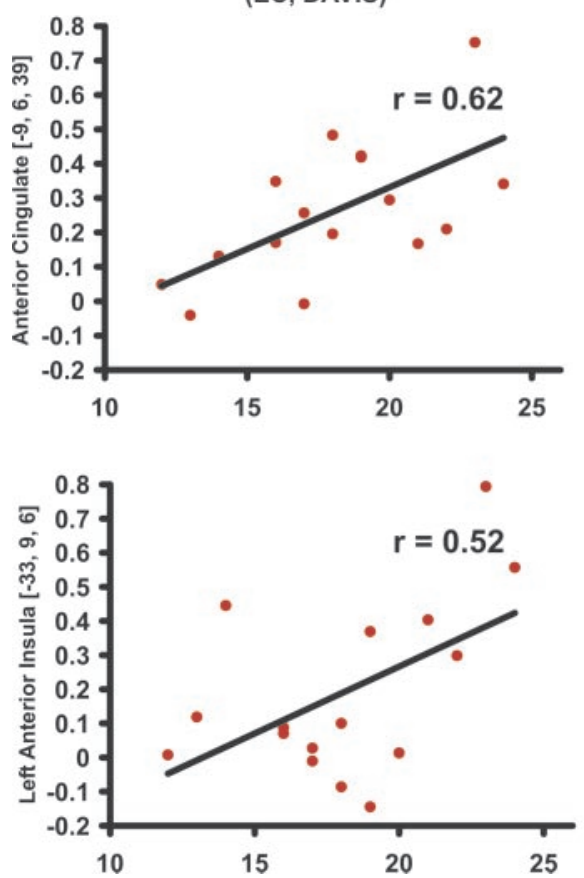

B

Balanced Emotional Empathy Scale (BEES, Mehrabian)
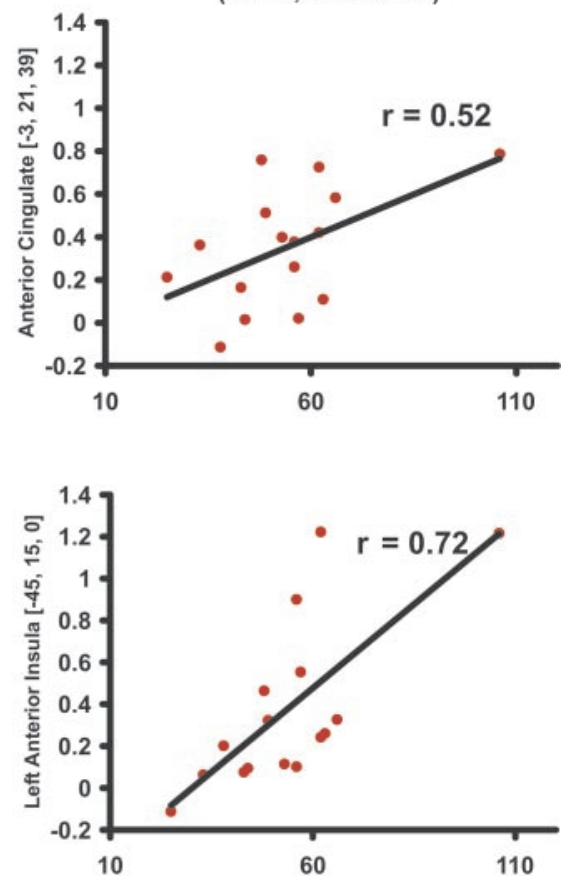

Fig. 4. Activation level (parameter estimates) observed within peaks of the ACC and the left insula during empathy-related conditions (pain-no pain in other) are significantly correlated with individual differences in empathy as measured by (A) the Empathic Concern Scale of Davis (20) and (B) the Balanced Emotional Empathy Scale of Mehrabian $(18,19)$. The lines represent the linear best fit; $r$ refers to the correlation coefficient. All correlations are significant on the $P<0.05$ level. Peak activations lie within regions of ACC and left insula that were activated in the simple contrast pain-no pain in others. Coordinates refer to peak activations and are in $\mathrm{mm}$.

condition of the body, similar to temperature, sensual touch, itch, hunger, or thirst $(25,33)$. More specifically, it is assumed that an image of the body's internal state is mapped to the brain by afferents that provide input by way of the ventromedial thalamic nucleus to area 3 a (sensorimotor cortex), as well as to the mid/posterior dorsal insula. In humans, this high-resolution, modality-specific sensory representation of the physiological condition of the body in the posterior insula is initially re-represented in the AI on the same side of the brain, and then, by way of a callosal pathway, remapped to the other side of the brain in the right AI. Such a second-order rerepresentation in the right $\mathrm{AI}$ is assumed to subserve subjective feelings and the awareness of a physical self as a feeling entity. At the same time, afferents also project by way of the medial dorsal thalamic nucleus to produce behavioral drive in ACC. Thus, direct activation of both the insula (limbic sensory cortex) and the ACC (the limbic motor cortex) may correspond to a simultaneous generation of both a feeling and an affective motivation with its attendant autonomic effects $(25,33)$. Indeed, imaging studies focusing on the relation between peripheral measures of arousal and brain activity give robust evidence for the crucial role of rostral ACC and AI cortices in the representation of internal bodily states of arousal, as well as emotional awareness $(26,27)$. Furthermore, activation in these regions has been observed in a wide range of imaging studies associated with positive and negative subjective feelings expressed by subjective ratings of facial trustworthiness (34), musical enjoyment (35), sensual touch (36), and distress resulting from social exclusion (37). More generally, these regions may play a critical role in second-order representations of bodily homeostatic states that underpin core representations of self $(38,39)$.

Our data suggest that empathizing with the pain of others does not involve the activation of the whole pain matrix, but is based on activation of those second-order rerepresentations containing the subjective affective dimension of pain. Accordingly, we propose that these cortical re-representations have a dual function. First, they form the basis for our ability to form subjective representation of feelings that allow us to predict the effects of emotional stimuli with respect to the self. Second, they serve as the neural basis for our ability to understand the emotional importance of a particular stimulus for another person and to predict its likely associated consequences. From a functional and evolutionary perspective, a detailed representation of the source and nature of a noxious stimulus (i.e., intensity, location) is of functional relevance when it concerns our own body, allowing effective engagement of executive action systems (i.e., removing the noxious source). In contrast, the understanding of someone else's emotional reaction to pain does not necessitate such a detailed sensorydiscriminative representation of the noxious stimulus but rather a representation of the subjective relevance of the stimulus as reflected in the subjective unpleasantness that the other person feels. Such decoupled representations - which are independent of the sensory inputs of the outside worldhave been postulated to be necessary for our ability to mentalize, that is, to understand the thoughts, beliefs, and intentions of others (2). Our data suggest that we use similar decoupled representations to understand the feelings of others and that our ability to empathize has evolved from a system for representing our internal bodily states and subjective feeling states.

References and Notes

1. D. Premack, G. Woodruff, Behav. Brain Sci. 1, 515 (1978).

2. U. Frith, C. D. Frith, Philos. Trans. R. Soc. London Ser. B 358, 459 (2003).

3. V. Gallese, Philos. Trans. R. Soc. London Ser. B $\mathbf{3 5 8}$, 517 (2003).

4. R. Adolphs, Trends Cognit. Sci. 3, 469 (1999).

5. R. Adolphs, Nature Rev. Neurosci. 4, 165 (2003).

6. L. Brothers, Concepts Neurosci. 1, 27 (1990).

7. R. W. Levenson, A. M. Ruef, J. Pers. Soc. Psychol. 63, 234 (1992).

8. S. D. Preston, F. B. M. de-Waal, Behav. Brain Sci. 25, 1 (2002).

9. W. Prinz, Eur. J. Cognit. Psychol. 9, 129 (1997).

10. S. K. Langer, An Essay on Human Feelings (John Hopkins Press, Baltimore, MD, 1988).

11. J. Decety, T. Chaminade, Neuropsychologia 41, 127 (2003).

12. L. Carr, M. lacoboni, M. C. Dubeau, J. C. Mazziotta, G. L. Lenzi, Proc. Natl. Acad. Sci. U.S.A. 100, 5497 (2003).

13. B. Wicker et al., Neuron 40, 655 (2003).

14. K. D. Davis, Neurol. Res. 22, 313 (2000).

15. R. Peyron, B. Laurent, L. Garcia-Larrea, Neurophysiol. Clin. 30, 263 (2000).

16. Materials and methods are available as supporting material on Science Online.

17. N. Picard, P. L. Strick, Cerebral Cortex 6, 342 (1996).

18. A. Mehrabian, N. Epstein, J. Pers. 40, 525 (1972)

19. A. Mehrabian, Aggress. Behav. 23, 433 (1997).

20. M. A. Davis, JSAS Catalog of Selected Documents in Psychology 10, 85 (1980) (American Psychological Association, Journal Supplement Abstract Service).

21. W. D. Hutchison, K. D. Davis, A. M. Lozano, R. R. Tasker, J. O. Dostrovsky, Nature Neurosci. 2, 403 (1999).

22. J. Grezes, J. Decety, Hum. Brain Mapp. 12, 1 (2001). 23. U. Bingel et al., Neuroimage 18, 740 (2003).

24. J. C. Brooks, T. J. Nurmikko, W. E. Bimson, K. D. Singh, N. Roberts, Neuroimage 15, 293 (2002).

25. A. D. Craig, Nature Rev. Neurosci. 3, 655 (2002).

26. H. D. Critchley, S. Wiens, P. Rotshtein, A. Öhman, R. J. Dolan, Nature Neurosci. 7, 189 (2004).

27. H. D. Critchley et al., Brain 126, 2139 (2003).

28. P. Rainville, G. H. Duncan, D. D. Price, B. Carrier, M. C. Bushnell, Science 277, 968 (1997).

29. R. Peyron et al., Brain 122, 1765 (1999). 
30. A. D. Craig, K. Chen, D. Bandy, E. M. Reiman, Nature Neurosci. 3, 184 (2000).

31. A. Ploghaus et al., Science 284, 1979 (1999).

32. P. Petrovic, E. Kalso, K. M. Petersson, M. Ingvar, Science 295, 1737 (2002).

33. A. D. Craig, Curr. Opin. Neurobiol. 13, 500 (2003).

34. J. S. Winston, B. A. Strange, J. O'Doherty, R. J. Dolan, Nature Neurosci. 5, 277 (2002).

35. A. J. Blood, R. J. Zatorre, Proc. Natl. Acad. Sci. U.S.A 98, 11818 (2001).

36. H. Olausson et al., Nature Neurosci. 5, 900 (2002).
37. N. I. Eisenberger, M. D. Lieberman, K. D. Williams, Science 302, 290 (2003).

38. P. S. Churchland, Science 296, 308 (2002).

39. A. R. Damasio, Descartes' Error (Putnam, New York, 1994).

40. This work was supported by a personal grant for T.S from the German Academy of Natural Sciences Leopoldina, Halle, with the grant BMBF-LPD 9901/873 from the Ministry of Education and Science, by the Wellcome Department of Imaging Neuroscience, and by the University College of London. R.J.D. and C.D.F. are in receipt of Wellcome Trust program grants. We thank S. Kiebel, J. Schultz, K. Wiech, R. Kalisch, P. Aston, E. Featherstone, and P. Allen for their help.

Supporting Online Material

www.sciencemag.org/cgi/content/full/303/5661/1157/ DC1

Materials and Methods

Fig. S1

Tables S1 to S5

References

11 November 2003; accepted 23 December 2003

\section{Placebo-Induced Changes in fMRI in the Anticipation and Experience of Pain}

\author{
Tor D. Wager, ${ }^{1 *_{\dagger} \dagger}$ James K. Rilling, ${ }^{2}$ Edward E. Smith, ${ }^{1}$ \\ Alex Sokolik, ${ }^{3}$ Kenneth L. Casey, ${ }^{3}$ Richard J. Davidson, ${ }^{4}$ \\ Stephen M. Kosslyn, ${ }^{5}$ Robert M. Rose, ${ }^{6}$ Jonathan D. Cohen ${ }^{2,7}$
}

\begin{abstract}
The experience of pain arises from both physiological and psychological factors, including one's beliefs and expectations. Thus, placebo treatments that have no intrinsic pharmacological effects may produce analgesia by altering expectations. However, controversy exists regarding whether placebos alter sensory pain transmission, pain affect, or simply produce compliance with the suggestions of investigators. In two functional magnetic resonance imaging (fMRI) experiments, we found that placebo analgesia was related to decreased brain activity in pain-sensitive brain regions, including the thalamus, insula, and anterior cingulate cortex, and was associated with increased activity during anticipation of pain in the prefrontal cortex, providing evidence that placebos alter the experience of pain.
\end{abstract}

The idea that sensory experience is shaped by one's attitudes and beliefs has gained currency among psychologists, physicians, and the general public. Perhaps nowhere is this more apparent than in our ability to modulate pain perception. A special case of this phenomenon is placebo analgesia, in which the mere belief that one is receiving an effective analgesic treatment can reduce pain (1-5). Recently, some researchers have attributed placebo effects to response bias and/or to publication biases (6), which raises the issue of whether placebo treatments actually influence the sensory, affec-

${ }^{1}$ Department of Psychology, University of Michigan, 525 East University, Ann Arbor, MI 481091109 , USA. ${ }^{2}$ Center for the Study of Brain, Mind and Behavior, Princeton University, Princeton, NJ 08544, USA. ${ }^{3}$ Department of Neurology, Veterans Affairs Medical Center, University of Michigan, Ann Arbor, MI 48109, USA. ${ }^{4}$ Department of Psychology, University of Wisconsin, Madison, WI 53706, USA. ${ }^{5}$ Department of Psychology, Harvard University, Cambridge, MA 02138, USA. ${ }^{2}$ Mind Brain Body and Health Initiative, University of Texas Medical Branch, Galveston, TX 77555, USA. ${ }^{7}$ Department of Psychology, Princeton University, Princeton, NJ 08544, USA.

*To whom correspondence should be addressed. E-mail: torw@umich.edu, tor@paradox.psych.columbia.edu

$\dagger$ Present address: Department of Psychology, Columbia University, 1190 Amsterdam Avenue, New York, NY 10027, USA. tive, and cognitive processes that mediate the experience of pain.

One important piece of evidence that placebo effects are not simply due to response or publication bias is that such effects can be reversed by the mu-opioid antagonist naloxone $(2,3,7)$, suggesting that some kinds of placebo effects may be mediated by the opioid system. However, naloxone has also been shown to produce hyperalgesia independent of placebo, in some cases offsetting rather than blocking the effects of placebo analgesia (8). Although pharmacological blockade provides suggestive evidence regarding the neurochemical mechanisms mediating placebo effects, such data do not illuminate the nature of the information-processing system that gives rise to such effects. Neuroimaging data can provide complementary evidence of how pain processing in the brain is affected by placebos and about the time course of pain processing. Identifying placebo-induced changes in brain activity in regions associated with sensory, affective, and cognitive pain processing (9) may provide insight into which components of pain processing are affected by placebo. In addition, identifying changes that occur at particular times - in anticipation of pain, early or late during pain processing - may shed light on how cognitive systems mediating expectancy interact with pain and opioid systems.

In two functional magnetic resonance imaging (fMRI) experiments $(n=24$ and $n=23$ ), we examined two hypotheses regarding the psychological and neural mechanisms that underlie placebo analgesia. Our first hypothesis was that if placebo manipulations reduce the experience of pain, pain-responsive regions of the brain should show a reduced fMRI blood oxygen leveldependent (BOLD) signal (a measure related to neural activity) during pain. [Painresponsive regions, or the "pain matrix," include thalamus, somatosensory cortex, insula, and anterior cingulate cortex (1014).] Our second hypothesis was that placebo modulates activity of the pain matrix by creating expectations for pain relief, which in turn inhibit activity in painprocessing regions. Converging evidence suggests that the prefrontal cortex (PFC), the dorsolateral aspect (DLPFC) in particular, acts to maintain and appropriately update internal representations of goals and expectations, which modulate processing in other brain areas $(15,16)$. Thus, stronger PFC activation during the anticipation of pain should correlate with greater placeboinduced pain relief as reported by participants and greater placebo-induced reductions in neural activity within pain regions (17).

Placebo reduces reported pain and brain activity in Study $\mathbf{1}$ (shock pain). The design of Study 1 is illustrated in Fig. 1A (see the figure legend for a description) (18). First, to confirm that application of shock elicited a neural response in painrelated areas, we compared brain activity in the intense shock versus no shock conditions. This revealed activation of the classic pain matrix $(11,14,19,20)$, including thalamus, primary somatosensory cortex/ primary motor cortex (S1/M1), secondary somatosensory cortex (SII), midbrain, anterior insula, anterior cingulate cortex (ACC), ventrolateral prefrontal cortex, and cerebellum (fig. S1). As expected, activations in thalamus, S1, SII, and M1 were larger in the left hemisphere, contralateral to the wrist where shocks were applied, whereas cerebellar activation was ipsilateral, although some bilateral activation was observed in each of these areas. We also 